\title{
Arachnostega gastrochaenae BERTLING, 1992 EN FÓSILES DEL DOBROTIVIENSE (ORDOVÍCICO MEDIO) DE LOS MONTES DE TOLEDO (SO DE ESPAÑA)
}

\author{
Nuno P.C. RODRIGUES $S^{1,2}$, María Dolores GIL \\ CID $^{1}$, Fernando ARROYO ${ }^{1}$, Miguel HUINEMAN ${ }^{1}$, \\ Ricardo LARA ${ }^{1}$ y Angélica TORICES ${ }^{1}$
}

\begin{abstract}
${ }^{1}$ Departamento de Paleontología e Instituto de Geología Económica (C.S.I.C.U.C.M.), Facultad de Ciencias Geológicas, Universidad Complutense de Madrid, Ciudad Universitaria, 28040 Madrid, España. E-mail: gilcid@geo.ucm.es.

${ }^{2}$ Grupo PALEO - Grupo de Paleontologia do Museu Nacional de História Natural, Rua da Escola Politécnica, nº 58, P-1294 Lisboa Codex, Portugal.
\end{abstract}

Rodrigues, N.P.C., Gil Cid, M.D., Arroyo, F., Huineman, M., Lara, R. \& Torices, A. 2005. Arachnostega gastrochaenae Bertling, 1992 en fósiles del Dobrotiviense (Ordovícico Medio) de los Montes de Toledo (SO de España) [Arachnostega gastrochaenae Bertling, 1992 on Dobrotivian (Middle Ordovician) fossils from Montes de Toledo (SW Spain)]. Revista Española de Paleontología, 20 (1), 57-64. ISSN 0213-6937.

\begin{abstract}
In the lower Dobrotivian (Middle Ordovician) of Montes de Toledo there are evidences of bioturbation in internal moulds of molluscs (bivalves, gastropods, rostroconchs and cephalopods), trilobites and echinoderms. They allow recognizing the presence of the organisms that left those evidences in the fossil record, increasing therefore the knowledge of Ordovician marine communities from SW Spain. The analysis of fossils where these structures are found allow us to infer that they were produced in a firm substrate (internal casts without evidences of post-diagenetical reworking). On the other hand, they have a type of preservation in negative and full relieves. Both observations suggest that they are bioturbation structures. The ichnotaxonomic study allows us to identify the specimens analysed as Arachnostega gastrochaenae. The interest of this discovery is related with the fact of being the first description of bioturbation structures in internal moulds of invertebrates from the Palaeozoic of Spain. A complete description of this ichnospecies makes possible to confirm its presence in siliciclastic facies. Also, it corroborates the antiquity of these ichnofossils. So, its stratigraphical record includes Ordovician, Carboniferous?-Permian?, Jurassic, Cretaceous and Recent. In the same way, the number of organism groups whose internal moulds present this type of bioturbation structures is increased, since up to now the only references were in bivalves, brachiopods, trilobites and, more recently, echinoderms. The producers of these burrows were probably vagile polychaetes or small crustaceans.
\end{abstract}

Key words: Arachnostega gastrochaenae, bioturbation structures, ichnofossils, Middle Ordovician, SW Spain.

\section{RESUMEN}

En el Dobrotiviense inferior (Ordovícico Medio) de los Montes de Toledo se encuentran evidencias de bioturbación en moldes internos de moluscos (bivalvos, gasterópodos, rostroconchas y cefalópodos), trilobites y equinodermos. De este modo, podemos identificar la presencia de los organismos que han dejado esas evidencias en el registro fósil, aumentando así el conocimiento sobre las comunidades marinas ordovícicas del SO español. El análisis de los fósiles donde se encuentran estas estructuras nos permite inferir que han sido producidas en un sustrato firme (moldes internos sin evidencias de reelaboración post-diagenética). Por otro lado, tienen un tipo de preservación en relieve negativo y completo. Ambas observaciones sugieren que son estructuras de bioturbación. El estudio icnotaxonómico nos permite llegar a la conclusión de que los ejemplares analizados se pueden identificar como Arachnostega gastrochaenae. El interés de este hallazgo está relacionado con la primera descripción de estructuras de bioturbación en moldes internos de invertebrados del Paleozoico español. La completa descripción de esta icnoespecie posibilita confirmar su presencia en facies siliciclásticas. Además, viene a corroborar 
la antigüedad de estos icnofósiles. Así, su registro estratigráfico incluye el Ordovícico, Carbonífero?-Pérmico?, Jurásico, Cretácico y Actualidad. Del mismo modo, viene a ampliar el número de grupos de organismos cuyos moldes internos presentan este tipo de estructuras de bioturbación, pues sólo se conocían ejemplos, hasta ahora, en bivalvos, braquiópodos, trilobites y, muy recientemente, equinodermos. Los organismos productores de estas galerías serían probablemente poliquetos vágiles o pequeños crustáceos.

Palabras clave: Arachnostega gastrochaenae, estructuras de bioturbación, icnofósiles, Ordovícico Medio, SO de España.

\section{INTRODUCCIÓN Y OBJETIVOS}

El presente trabajo consiste en mostrar una serie de ejemplos de estructuras de bioturbación conservadas en fósiles del Dobrotiviense inferior (Ordovícico Medio) de los Montes de Toledo (SO de España).

Las muestras estudiadas proceden de Navas de Estena (Ciudad Real; Fig. 1) y se ubican en el conjunto de formaciones conocido con el nombre general de "Capas con tristani", que incluye unidades muy potentes de pizarras, intercaladas con otras de cuarcitas, de menor espesor (Gutiérrez-Marco \& Rábano, 1999). En un contexto geológico más específico, podemos afirmar que estas muestras se encuentran en las pizarras negras de la unidad Pizarras de Navas de Estena (yacimiento NE-VIIc), que corresponde a la parte más baja de la Biozona Tournemini, es decir, la Sub-biozona Macrophtalma-Toledana (Rábano, 1990).

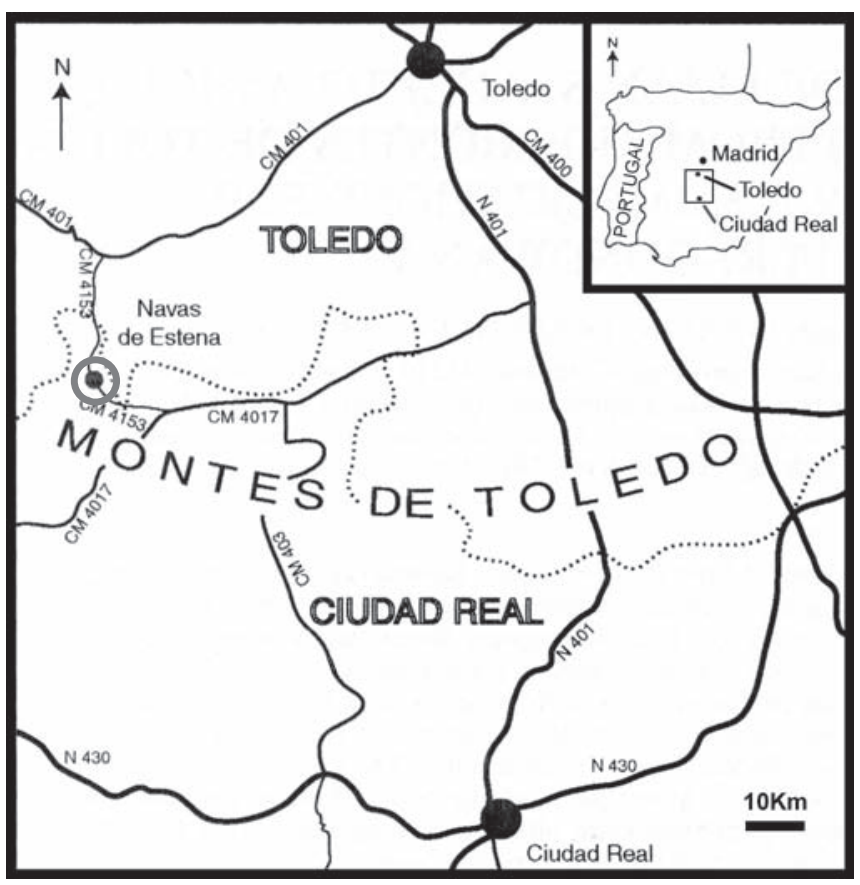

Figura 1. Localización geográfica de las muestras estudiadas (modificado de Ausich et al., 2002).

Geographical location of the studied samples (modified from Ausich et al., 2002).
A partir del análisis de los icnofósiles observados podemos identificar la presencia de los organismos que han dejado esas evidencias en el registro fósil, aumentando así el conocimiento sobre las comunidades marinas ordovícicas del SO español. Estas estructuras aparecen conservadas en los moldes internos de moluscos (bivalvos, gasterópodos, rostroconchas y cefalópodos), trilobites y equinodermos, asociación ésta característica de un ambiente de plataforma externa.

\section{ICNOLOGÍA SISTEMÁTICA}

$$
\text { Icnogénero Arachnostega Bertling, } 1992
$$

Icnoespecie tipo: Arachnostega gastrochaenae Bertling, 1992.

Diagnosis: Red de galerías alargadas, irregulares y ramificadas en el relleno sedimentario de conchas. Visibles sobre la superficie de moldes internos. El tamaño de la malla puede variar desde micrómetros hasta centímetros, dependiendo de la dimensión de la concha portadora y del organismo productor de las galerías (Bertling, 1992: 179; Damborenea \& Manceñido, 1996: 113; Aceñolaza et al., 2003: 318).

\section{Arachnostega gastrochaenae Bertling, 1992} Figs. 2a-h y $3 a-h$

1992 Arachnostega gastrochaenae Bertling, 180-182, fig. 2a-f. 1994 Arachnostega gastrochaenae Bertling; Fürsich et al., 161, lám. 3, figs. 1, 2 y 4.

1996 Arachnostega isp. aff. gastrochaenae Bertling; Damborenea y Manceñido, 113, lám. 1, figs. 1, 3 y 5; fig. 1a-b.

2002 Arachnostega; Rodrigues et al., fig. 1A-C.

2002 Arachnostega gastrochaenae Bertling; Aceñolaza y Aceñolaza, fig. 6D.

2003 Arachnostega gastrochaenae Bertling; Aceñolaza et al., 318-320, fig. 4A-H.

Material: Numerosas muestras de moldes internos de bivalvos, gasterópodos, rostroconchas, cefalópodos, trilobites y equinodermos que contienen redes de galerías identificadas como Arachnostega gastrochaenae, procedentes del Dobrotiviense inferior (Ordovícico Medio) de Navas de Estena (Ciudad Real, SO de España). En concreto, en este artículo son figuradas 6 muestras 
depositadas en el Museu Nacional de História Natural da Universidade de Lisboa (Portugal), con las siglas MNHN-UL.II.418 a MNHN-UL.II.423, y otras 2 pertenecientes a la Colección Gil Cid, depositadas en el Departamento de Paleontología de la Facultad de Ciencias Geológicas de la Universidad Complutense de Madrid (España), con las siglas NE-VIIc-2008 y NE-VIIc-2012. Todas las muestras proceden del yacimiento NE-VIIc.

Diagnosis: Galerías ramificadas sobre la superficie de moldes internos, ovaladas en sección transversal, aumentando lentamente de diámetro por un factor de 5 a 10, aproximadamente. En las ramificaciones, la galería principal no está reducida en tamaño. Las galerías laterales, generalmente, tienen un diámetro menor. Las áreas poligonales sobre la superficie de moldes internos pueden ser producidas por la unión de galerías ramificadas (Bertling, 1992: 180).

Descripción: Red de galerías alargadas, irregulares y ramificadas, producidas sobre moldes internos de bivalvos, gasterópodos, rostroconchas, cefalópodos, trilobites y equinodermos. Las galerías son ovaladas a redondeadas en sección transversal. El diámetro de cada galería oscila, aproximadamente, entre 0,1 y $0,5 \mathrm{~mm}$, dependiendo de su intersección con la superficie del molde. Debido a que cada estructura es una red de galerías que se puede prolongar hacia el interior del molde interno, a veces en la superficie de éste las galerías parecen tener una morfología simple, punteada o lineal, aunque en realidad se observe la tendencia a ramificarse hacia el interior del sustrato (molde interno). El ángulo de ramificación puede variar entre $60^{\circ}$ y $120^{\circ}$, aproximadamente, llegando incluso a formarse auténticas redes de galerías anastomosadas o reticuladas. En los puntos de bifurcación se observa un ligero ensanchamiento de las galerías, debido a la unión de las mismas.

Discusión: El icnogénero Arachnostega es, hasta el momento, monoicnoespecífico, estando constituido por la icnoespecie Arachnostega gastrochaenae.

En este trabajo se pone en sinonimia la icnoespecie que Damborenea \& Manceñido (1996) identificaron como Arachnostega isp. aff. gastrochaenae, ya que los argumentos utilizados por estos autores para diferenciar sus ejemplares de los de Bertling (1992) no han tenido en cuenta las sugerencias de Pickerill (1994). Según este autor, sólo las diferencias en la dimensión de los icnofósiles, sin otras discrepancias en sus características, no son suficientes para distinguir una icnoespecie, ni tampoco debe ser motivo de dudas en cuanto a su identificación, no haciendo falta el término affinis. El propio Bertling (1992) indica que la distinción entre las posibles nuevas icnoespecies de Arachnostega no debe basarse sólo en la dimensión, sino que las galerías deben mostrar diferentes patrones de crecimiento en el tamaño o en las ramificaciones. Por otro lado, las diferencias en la naturaleza de la fauna portadora de estas galerías tampoco se debe considerar para justificar esas dudas.

Los dos ejemplares designados como Arachnostega isp. y figurados en el trabajo de Damborenea \& Manceñido
(1996) no entran en sinonimia en este artículo, al considerarse que son actuales. Uno de esos ejemplares aparece en las llanuras mareales de Jade Bay (Mar del Norte, Alemania) y consiste en galerías producidas por los poliquetos Nereis diversicolor Müller y Heteromastus filiformis Claparède en un molde interno del bivalvo actual Mya arenaria Linné (Reineck, 1980). El otro ejemplar corresponde a un molde interno del bivalvo actual Geukensia demissa (Dillwyn), que está bioturbado por el poliqueto Nereis succinea (Frey \& Leuckart), no habiendo dudas cuanto al productor de estas estructuras que aparecen en las llanuras mareales de Sapelo Island (costa de Georgia, Estados Unidos), como se puede observar en Basan \& Frey (1977). Estos autores presentan otro ejemplo actual de igual procedencia, aunque esté producido por el crustáceo decápodo Uca pugnax (Smith), constituyendo una evidencia dada por la Neoicnología de como estructuras semejantes pueden estar producidas por diferentes organismos.

Los icnofósiles descritos en este trabajo se distinguen de Korymbichnus conflabellatus Damborenea \& Manceñido, 1996 (otra icnoespecie que también consiste en galerías producidas en moldes internos) por poseer ramificaciones más irregulares y segmentos de galerías relativamente más cortos, que suelen presentarse anastomosados o reticulados.

En el artículo de Rodrigues et al. (2002) se hacen unas primeras observaciones que han dado origen al presente trabajo, aunque los ejemplares se hayan clasificado sólo a nivel icnogenérico. El análisis más detallado de los icnofósiles ha permitido confirmar que se trataban de Arachnostega gastrochaenae.

Distribución: La icnoespecie Arachnostega gastrochaenae ha sido descrita en el Arenig inferior e medio (Ordovícico Inferior) de Argentina (Aceñolaza et al., 2003), Dobrotiviense inferior (Ordovícico Medio) de España (este trabajo), Portlandiense superior (Jurásico Superior) de Inglaterra (Fürsich et al., 1994), Oxfordiense superior (Jurásico Superior) de Alemania (Bertling, 1992) y Calloviense inferior (Jurásico Medio) de Argentina (Damborenea \& Manceñido, 1996). Por otro lado, existen también referencias en el Arenig-Llanvirn? (Ordovícico InferiorMedio?) de Argentina (Aceñolaza \& Aceñolaza, 2002), Dobrotiviense inferior (Ordovícico Medio) de España (Gutiérrez-Marco \& Rábano, 1999; Gutiérrez-Marco et al., 1999; Rodrigues et al., 2002; Herranz Araújo et al., 2003), Llanvirn (Ordovícico Medio) de la República Checa (Bruthansová \& Kraft, 2003), Carbonífero Superior?-Pérmico Inferior? de Uruguay (Sprechmann et al., 2001) y Campaniense-Maastrichtiense (Cretácico Superior) de Holanda y Bélgica (Donovan \& Jagt, 2002), aunque en todos estos casos no se hagan descripciones detalladas. Existen ejemplos actuales de estructuras semejantes a esta icnoespecie procedentes de Sapelo Island (costa de Georgia, Estados Unidos; Basan \& Frey, 1977) y Jade Bay (Mar del Norte, Alemania; Reineck, 1980). 

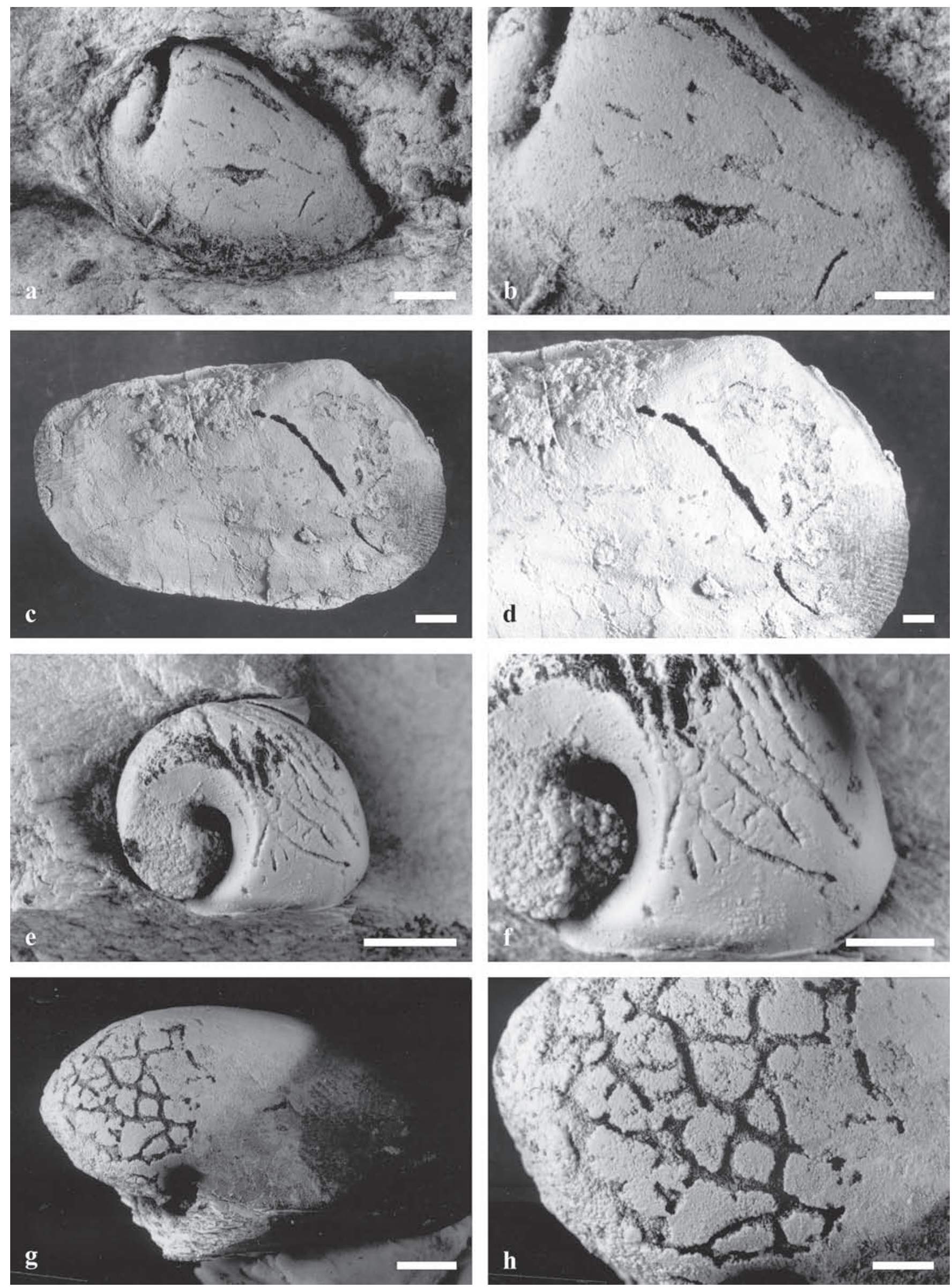


\section{¿BIOTURBACIÓN O BIOEROSIÓN?}

La realización de un estudio sobre evidencias de bioturbación o de bioerosión necesita una aclaración previa sobre lo que se entiende por estos términos. Fernández López (2000: 142 y 143) define bioturbación como la "alteración de un sedimento blando o firme debido a la remoción provocada por organismos" y bioerosión como la "acción bioerosiva o eliminación de materiales de un sustrato duro por la acción directa de los organismos" (véanse también las definiciones clásicas dadas por Bromley, 1970).

Para que se pueda entender si estamos en presencia de bioturbación o de bioerosión tenemos, por tanto, que analizar el tipo de sustrato donde se encuentran conservados estos icnofósiles. Mayoral (2001) indica que las estructuras bioerosivas pueden ser producidas en distintos tipos de sustrato duro tales como las rocas, superficies endurecidas y restos esqueléticos de organismos. Este autor indica para el Paleozoico Inferior, y especialmente para el Ordovícico, la existencia de estructuras bioerosivas constituidas por perforaciones de organismos endolíticos (bacterias y talofitas), briozoos ctenostomados, esponjas, bivalvos y sipuncúlidos. Además, hace referencia a casos de depredación y/o parasitismo en esta época.

Sin embargo, el análisis de nuestros ejemplares nos permite verificar que se tratan de galerías producidas en moldes internos. Estas galerías aparecen en la superficie de los moldes internos, prolongándose hacia su interior. Por eso, las estructuras analizadas no podrían ser contramoldes de organismos incrustantes, pues estos son epilíticos.

Las muestras estudiadas presentan una completa disolución de los restos esqueléticos, lo que no permite la conservación de posibles evidencias de bioerosión en la concha. Las observaciones tafonómicas nos indican que no hubo reelaboración, ya que se observa la presencia de espacios vacíos correspondientes a las conchas disueltas entre los moldes internos y externos, no existe abrasión de los moldes y el sedimento del relleno es semejante al encajante. Por otro lado, muchos de los fósiles encontrados en la unidad estudiada son característicos, permitiendo saber la edad de los sedimentos que los contienen. Además, las estructuras que aparecen en los moldes jamás se observan en los sedimentos adyacentes, como sería de esperar en el caso de superficies colonizadas por litobiontes.
En consecuencia, podemos plantear la hipótesis de estar ante estructuras de bioturbación, ya que el sustrato colonizado por los organismos bioturbadores sería firme (moldes internos), no estando todavía completamente consolidado, aunque en Gil Cid et al. (2002) se pensara que se trataba de bioerosión. De hecho, en el registro fósil hay que tener cuidado para no confundir las galerías producidas pre-litificación con las perforaciones originadas post-litificación (cf. Boyd \& Newell, 1972; Basan \& Frey, 1977).

Un argumento más a favor de la bioturbación consiste en el hecho de que sería muy difícil que un organismo pudiera perforar dos sustratos reológicamente distintos (resto esquelético carbonatado y molde interno arcilloso) a la vez (Neto de Carvalho, com. pers.), lo que sugiere que estas estructuras se producen en el molde interno y no en el resto esquelético, constituyendo una red de pequeñas galerías.

Otro argumento de apoyo a la propuesta de evidencias de bioturbación consiste en el estudio del tipo de conservación que presentan las estructuras, observándose que todas ellas aparecen en relieve negativo y completo. Para que pudiéramos considerar la posible presencia de bioerosión en moldes internos, estas estructuras tendrían que estar conservadas en relieve positivo (como se observa, por ejemplo, en el icnogénero Entobia, cuando éste aparece en moldes internos; e.g., Bromley, 1970) o negativo (en el caso de haber reelaboración del molde interno y éste ser colonizado por organismos euendolíticos), y jamás en relieve completo.

\section{INTERPRETACIÓN Y CONCLUSIONES}

Basándonos en el modelo que Bertling (1992) utiliza para explicar la génesis de estas estructuras, podemos decir que se producen después de la muerte del organismo portador de las mismas y el posterior relleno sedimentario de su concha, formándose un sustrato firme, cohesivo, pero no totalmente consolidado (molde interno). Cuando el sustrato aún se encuentra firme es colonizado por los organismos bioturbadores, que construyen su red de galerías en el molde interno, en busca de materia orgánica con que alimentarse, constituyendo estructuras de tipo fodinichnia. El sustrato no podría estar totalmente blando

Figura 2. Arachnostega gastrochaenae Bertling, 1992 del Dobrotiviense inferior (Ordovícico Medio) de Navas de Estena (Ciudad Real, España). a-d, Ejemplares producidos en moldes internos de los bivalvos Redonia (a; MNHN-UL.II.418) y Coxiconcha (c; NE-VIIc-2012), y respectivos detalles (b y d). e-h, Ejemplares producidos en moldes internos de los gasterópodos Clathrospira (e; MNHN-UL.II.419) y Sinuites (g; MNHN-UL.II.420), y respectivos detalles (f y h). Escala = 4mm (en a, c, e y g) y $2 \mathrm{~mm}$ (en b, d, f y h).

Arachnostega gastrochaenae Bertling, 1992 of the lower Dobrotivian (Middle Ordovician) from Navas de Estena (Ciudad Real, Spain). a-d, Specimens produced in internal moulds of the bivalves Redonia ( $a$; MNHN-UL.II.418) and Coxiconcha ( $c$; $N E$-VIIc-2012), and respective details ( $b$ and d). $\boldsymbol{e}-\boldsymbol{h}$, Specimens produced in internal moulds of the gastropods Clathrospira (e; MNHN-UL.II.419) and Sinuites (g, MNHN-UL.II.420), and respective details ( $f$ and $h$ ). Scale bar = 4mm (in a, $c, e$ and $g$ ) and $2 \mathrm{~mm}$ (in $b, d$, $f$ and $h$ ). 

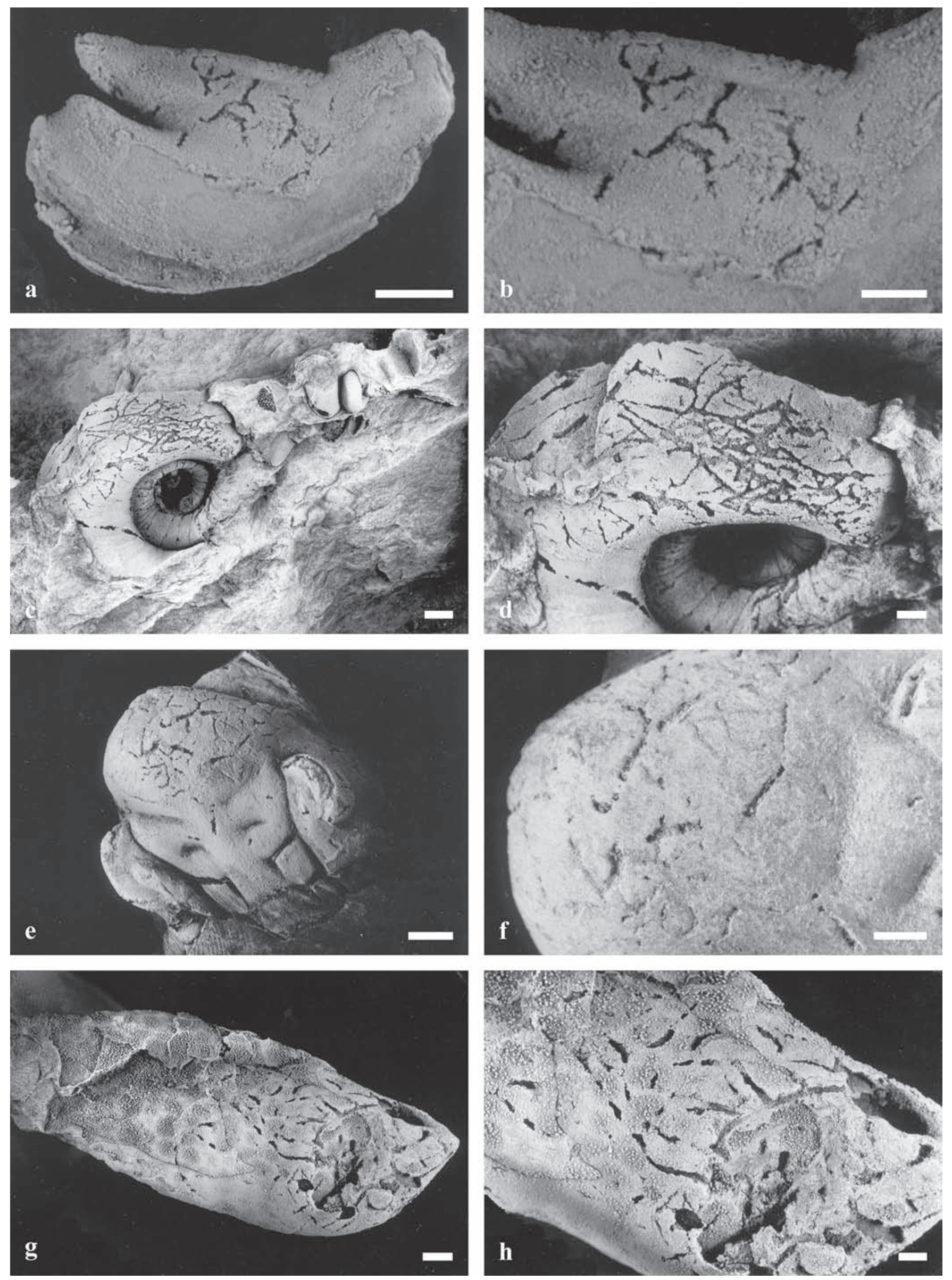
porque eso implicaría el desplome de las galerías después de ser producidas por el organismo excavador. Los productores de Arachnostega serían excavadores detritívoros o depositívoros y podrían ser poliquetos vágiles o pequeños crustáceos (Bertling, 1992; Damborenea \& Manceñido, 1996; Aceñolaza et al., 2003), si se compara con las estructuras que se producen en la actualidad en moldes internos (cf. Basan \& Frey, 1977; Reineck, 1980). Recientemente, Bruthansová \& Kraft (2003) hacen referencia a la presencia de icnofósiles tipo Arachnostega, asociados a peloides fecales que aparecen en trilobites y equinodermos, planteando la hipótesis de que estos peloides podrían ser producidos por los organismos bioturbadores responsables por la construcción de la red de galerías característica de este icnogénero.

Los ejemplares de Arachnostega descritos por Bertling (1992) aparecen en calizas y calizas margosas arrecifales. El material que aparece descrito en Fürsich et al. (1994) se encuentra, igualmente, en calizas características de arrecifes. Las estructuras de bioturbación descritas por Damborenea \& Manceñido (1996) fueron relacionadas con ambientes de plataforma carbonatada, interna y externa. Aceñolaza \& Aceñolaza (2002) señalan un ambiente de plataforma de aguas poco profundas para sus ejemplares encontrados en una secuencia siliciclástica. Los icnofósiles descritos en el trabajo de Aceñolaza et al. (2003) están ubicados en ambientes siliciclásticos de plataforma interna y de abanico deltaico con aporte volcaniclástico. Los ejemplos actuales aparecen en ambientes de llanura mareal, constituyendo sustratos cohesivos, pero no totalmente consolidados, de fangos y arenas fangosas (cf. Basan \& Frey, 1977; Reineck, 1980; Damborenea \& Manceñido, 1996; Aceñolaza et al., 2003). Las estructuras estudiadas en el presente trabajo se refieren a un ambiente caracterizado por un sustrato fangoso, encontrándose en litofacies de pizarras negras. Corresponde a un medio de plataforma externa siliciclástica, distinguiéndose así unas condiciones paleoambientales distintas a las referenciadas para los demás casos relacionados con este icnogénero. Por este motivo, no se debe relacionar Arachnostega con un ambiente específico, ya que puede aparecer tanto en facies someras como más profundas y tanto carbonatadas como siliciclásticas. El factor paleoambiental más importante en estos icnofósiles consiste en el hecho de que constituyen excelentes indicadores de sustratos fangosos firmes, cohesivos, pero no totalmente consolidados, encontrándose confinados a moldes internos de organismos, que constituyen un microhábitat (cf. Damborenea \& Manceñido, 1996).

En este trabajo se ha podido corroborar la antigüedad de la icnoespecie Arachnostega gastrochaenae, verificándose su existencia ya en el Paleozoico Inferior y llegando hasta la Actualidad, aunque su registro en el tiempo no sea continuo, teniendo en cuenta los datos conocidos hasta la fecha. El presente artículo constituye la primera descripción de Arachnostega gastrochaenae en el registro estratigráfico español, encontrándose, hasta el momento, sólo en el Ordovícico Medio.

La presente contribución permite también ampliar el número de grupos de organismos en cuyos moldes internos aparecen estas estructuras de bioturbación, ya que hasta la fecha sólo se habían encontrado en moldes internos de bivalvos, braquiópodos, trilobites (cf. Damborenea \& Manceñido, 1996; Gutiérrez-Marco \& Rábano, 1999) y, muy recientemente, equinodermos (Bruthansová \& Kraft, 2003), añadiéndose ahora gasterópodos, rostroconchas y cefalópodos. Esto nos permite confirmar la presencia de Arachnostega en moldes internos de organismos endobentónicos, semi-infaunales, epibentónicos y nectobentónicos (post-mortem y posterior al relleno sedimentario). La observación de las muestras estudiadas refleja que existe una clara tendencia por parte del productor de estas estructuras a preferir la colonización de moldes internos de moluscos.

\section{AGRADECIMIENTOS}

Los autores agradecemos las numerosas sugerencias de Carlos Neto de Carvalho (Centro de Geologia da Universidade de Lisboa y Grupo Paleo - Grupo de Paleontologia do Museu Nacional de História Natural). También queremos expresar nuestro reconocimiento a los revisores, el Dr. Jordi M. de Gibert y un revisor anónimo, por las sugerencias realizadas al manuscrito y que suponen una mejora en la claridad del texto.

Figura 3. Arachnostega gastrochaenae Bertling, 1992 del Dobrotiviense inferior (Ordovícico Medio) de Navas de Estena (Ciudad Real, España). a-b, Ejemplares producidos en un molde interno del rostroconcha Ribeiria (a; NE-VIIc-2008) y respectivo detalle (b). c-d, Ejemplares producidos en un molde interno del cefalópodo Curtoceras (c; MNHN-UL.II.421) y respectivo detalle (d). e-f, Ejemplares producidos en un molde interno del trilobites Eodalmanitina (e; MNHN-UL.II.422) y respectivo detalle (f). g-h, Ejemplares producidos en un molde interno del equinodermo Calix (g; MNHN-UL.II.423) y respectivo detalle (h). Escala $=4 \mathrm{~mm}$ (en a, c, e y g) y $2 \mathrm{~mm}$ (en b, d, f y h).

Arachnostega gastrochaenae Bertling, 1992 of the lower Dobrotivian (Middle Ordovician) from Navas de Estena (Ciudad Real, Spain). $\boldsymbol{a}-\boldsymbol{b}$, Specimens produced in an internal mould of the rostroconch Ribeiria (a; NE-VIIc-2008) and respective detail (b). $\boldsymbol{c}$-d, Specimens produced in an internal mould of the cephalopod Curtoceras (c; MNHN-UL.II.421) and respective detail $(d) . e-f$, Specimens produced in an internal mould of the trilobite Eodalmanitina (e; MNHN-UL.II.422) and respective detail $(f) . g-h$, Specimens produced in an internal mould of the echinoderm Calix ( $g$; MNHN-UL.II.423) and respective detail (h). Scale bar $=4 m m$ (in $a, c, e$ and $g$ ) and $2 m m$ (in $b, d$, f and $h$ ). 


\section{BIBLIOGRAFÍA}

Aceñolaza, G. \& Aceñolaza, F.G. 2002. Ordovician Trace Fossils of Argentina. In: Aspects of the Ordovician System in Argentina (Ed. F.G. Aceñolaza). Instituto Superior de Correlación Geológica, Tucumán, Serie de Correlación Geológica, 16, 177-193.

Aceñolaza, G.F., Gutiérrez-Marco, J.C. \& Peralta, S. 2003. Arachnostega gastrochaenae Bertling (traza fósil) en las secuencias volcaniclásticas de la Formación Suri, Sistema de Famatina, Argentina. Ameghiniana, 40, 315-322.

Ausich, W.I., Gil Cid, M.D. \& Domínguez Alonso, P. 2002. Ordovician [Dobrotivian (Llandeillian stage) to Ashgill] crinoids (phylum Echinodermata) from the Montes de Toledo and Sierra Morena, Spain with implications for paleogeography of peri-Gondwana. Journal of Paleontology, 76, 975-992.

Basan, P.B. \& Frey, R.W. 1977. Actual-palaeontology and neoichnology of salt marshes near Sapelo Island, Georgia. In: Trace fossils 2 (Eds. T.P. Crimes \& J.C. Harper). Seel House Press, Liverpool, Geological Journal Special Issue, 9, 41-70.

Bertling, M. 1992. Arachnostega n. ichnog. - burrowing traces in internal moulds of boring bivalves (Late Jurassic, northern Germany). Paläontologische Zeitschrift, 66, 177-185.

Boyd, D.W. \& Newell, N.D. 1972. Taphonomy and diagenesis of a Permian fossil assemblage from Wyoming. Journal of Paleontology, 46, 1-14.

Bromley, R.G. 1970. Borings as trace fossils and Entobia cretacea Portlock, as an example. In: Trace fossils (Eds. T.P. Crimes \& J.C. Harper). Seel House Press, Liverpool, Geological Journal Special Issue, 3, 49-90.

Bruthansová, J. \& Kraft P. 2003. Pellets independent of or associated with Bohemian Ordovician body fossils. Acta Palaeontologica Polonica, 48, 437-445.

Damborenea, S.E. \& Manceñido, M.O. 1996. Icnofósiles (Nucleocavia) preservados sobre moldes internos de conchillas del Jurásico Medio del oeste argentino. Asociación Paleontológica Argentina, Publicación Especial, 4, 111-120.

Donovan, S.K. \& Jagt, J.W.M. 2002. Ichnology of the type area of the Maastrichtian Stage (Upper Cretaceous): burrowing and boring immediately prior to the $\mathrm{K} / \mathrm{T}$ boundary event. In: Abstracts of the 46th Annual Meeting of the Palaeontological Association (Ed. D.A.T. Harper). University of Cambridge, Newsletter, 51, 89.

Fernández López, S.R. 2000. Temas de Tafonomía. Departamento de Paleontología, Facultad de Ciencias Geológicas, Universidad Complutense de Madrid, 1-167.

Fürsich, F.T., Palmer, T.J. \& Goodyear, K.L. 1994. Growth and disintegration of bivalve-dominated patch reefs in the Upper Jurassic of southern England. Palaeontology, 37, 131-171.

Gil Cid, M.D., Arroyo, F., Lara, R., Rodrigues, N.P.C. \& Torices, A. 2002. Taphonomic features on the marine assemblages in the Lower Palaeozoic (Cambrian-Ordovician) from the SW of Spain. In: Current Topics on Taphonomy and Fossilization (Eds. M. De Renzi, M.V. Pardo Alonso, M. Belinchón, E. Peñalver, P. Montoya \& A. MárquezAliaga). Ajuntament de Valencia, 257-267.

Gutiérrez-Marco, J.C. \& Rábano, I. 1999. Fósiles del Neoproterozoico y Paleozoico Inferior de Castilla-la-Mancha. In: La huella del pasado: fósiles de Castilla-la-Mancha (Coords. E. Aguirre \& I. Rábano). Junta de Comunidades de Castilla-la-Mancha, Toledo, Patrimonio Histórico-Arqueología: Castilla-la-Mancha, 16, 25-50.

Gutiérrez-Marco, J.C., Aramburu, C., Arbizu, M., Bernárdez, E., Hacar Rodríguez, M.P., Méndez-Bedia, I., Montesinos López, R., Rábano, I., Truyols, J. \& Villas, E. 1999. Revisión bioestratigráfica de las pizarras del Ordovícico Medio en el noroeste de España (zonas Cantábrica, Asturoccidental-leonesa y Centroibérica septentrional). Acta Geologica Hispanica, 34, 3-87.

Herranz Araújo, P., Gutiérrez-Marco, J.C., Pieren Pidal, A.P., Robardet, M., San José Lancha, M.A., Rábano, I. \& Sarmiento, G.N. 2003. The Ordovician succession from the western Iberian Ranges (NE Spain): a review with new data. In: Ordovician from the Andes (Eds. G.L. Albanesi, M.S. Beresi \& S.H. Peralta). Instituto Superior de Correlación Geológica, Tucumán, Serie de Correlación Geológica, 17, 417-424.

Mayoral, E. 2001. Evidencias de bioerosión en fósiles marinos del Paleozoico. In: La Era Paleozoica. El desarrollo de la vida marina - VII Jornadas Aragonesas de Paleontología (eds. J.A. Gámez Vintaned \& E. Liñán). Institución Fernando El Católico, Zaragoza, 223-249.

Pickerill, R.K. 1994. Nomenclature and taxonomy of invertebrate trace fossils. In: The Palaeobiology of Trace Fossils (Ed. S.K. Donovan). John Wiley \& Sons, Chichester, 3-42.

Reineck, H.E. 1980. Steinkerne in der Entstehung. Natur und Museum, 110, 44-47.

Rábano, I. 1990. Trilobites del Ordovícico Medio del sector meridional de la zona Centroibérica española. Publicaciones Especiales del Boletín Geológico y Minero, 1-233.

Rodrigues, N.P.C., Gil Cid, M.D., Arroyo, F., Huineman, M., Lara, R. \& Torices, A. 2002. Evidencias de bioturbación/ bioerosión en los fósiles del Dobrotiviense (Ordovícico Medio) de los Montes de Toledo (SW de España). In: Libro de Resúmenes de las XVIII Jornadas de la Sociedad Española de Paleontología y II Congreso Ibérico de Paleontología (Eds. J. Civis \& J.A. González Delgado). Universidad de Salamanca, 130-131.

Sprechmann, P., Silva, J., Gaucher, C., Montaña, J. \& Herrera, Z. 2001. Icnofósiles en concreciones de la Formación San Gregorio del Uruguay (Carbonífero Superior?-Pérmico Inferior?): implicancias paleoecológicas y paleoclimáticas. In: Resúmenes de la IV Reunión Argentina de Icnología y II Reunión de Icnología del Mercosur. Tucumán, 73.

Manuscrito recibido: 19 de Diciembre, 2002 Manuscrito aceptado: 21 de Febrero, 2005 